\title{
SU LA DIFFUSIONE DEGLI ELETTROLITI NEI COLLOIDI
}

\author{
Nota di LUIGI ROLLA').
}

1. Si abbia un cilindro di gelatina pura, e su due piani paralleli al piano di base e distanti fra loro di una quantità $2 a$, si trovino due elettrodi opportunamente preparati e provvisti di fori in modo da permettere facilmente il passaggio del colloide. Sul piano di base si faccia circolare dal tempo $t=-\%$ al tempo $t=\%$ una soluzione di un elettrolito (per es. $\mathrm{KCl}$ ), mantenuta cosi a una concentrazione costante $\mathrm{K}$. Trascorso il tempo $\theta$, si sostituisca immediatamente alla soluzione dell' acqua pura e si faccia circolare in modo che la concentrazione si conservi, sul piano limite, nulla.

La forza elettromotrice che si stabilirà fra i due elettrodi sarà una funzione delle concentrazioni e, precisamente, trattandosi di un elettrolito binario con ioni monovalenti,

$$
\mathrm{E}=\frac{\mathrm{R}}{\mathrm{F}} \mathrm{T} \frac{2 u}{u+v} \log _{e} \frac{c_{0}}{c_{1}}
$$

dove $\mathrm{R}$ è la costante dei gas; $\mathrm{T}$ è la temperatura assoluta; $\mathrm{F}=96 \check{0} 40 ; u, v$ sono le mobilità degli ioni e $c_{0}, c_{1}$ le concentrazioni rispettive dègli ioni nell' immediata vicinanza degli elettrodi.

La concentrazione $c$, alla distanza $x$ dal piano di base, e per il tempo $l$, è data da

') Lavoro eseguito nell' Istituto di Chimica generale della R. Universitả di Genova. 


$$
c=\frac{2 \mathrm{~K}}{\sqrt{\pi}} \int \begin{aligned}
& \overline{2 k \sqrt{\bar{t}-\overline{\bar{t}} / 2}} \\
& \frac{x}{\left.e^{-u^{2}} d u^{1}\right)} \\
& \frac{x}{2 k \sqrt{t+\bar{\theta} / 2}}
\end{aligned}
$$

dove $k^{2}$ è il coefficiente di diffusione nell' equazione di Fourier

$$
\frac{\partial c}{\partial t}=k^{2} \frac{\partial^{2} c}{\partial x^{2}}
$$

Svolgendo in serie di Taylor si trova

$$
c=\frac{2 K}{\overline{\sqrt{\pi}}}\left\{\theta \frac{\partial}{\partial t} \int_{\frac{x}{2 k \sqrt{t}}}^{\infty} e^{-u^{2}} d u+\frac{\theta^{3}}{24} \frac{\partial^{3}}{\partial t^{3}} \int_{\frac{x}{2 k \sqrt{t}}}^{\infty} e^{-u^{2}} d u+\ldots\right\}
$$

e dunque, se si trascurano $i$ termini di terzo ordine in $\theta$,

$$
c=\frac{2 K \theta}{\sqrt{\pi}} \frac{\partial}{\partial t} \int_{-\frac{x}{2 k \sqrt{t}}}^{\infty} e^{-u^{2}} d u
$$

Ciò premesso si osserverà che la f. e. m. fra i due punti del mezzo in cui sono posti gli elettrodi, diventerà massima col rapporto che intercede fra le loro concentrazioni. Per fissare le idee, poniamo che al primo elettrodo corrisponda la coordinata

$$
E+a
$$

e al secondo la coordinata

$$
\mathbb{E}-a
$$

1) Garbasso, Rend. R. Acc. dei Lincei, XX (5), to sem., fas. $4^{0}$ (1911). 
il rapporto, che si deve rendere massimo, sarà

$$
P=-\left(\frac{c+a \frac{\partial c}{\partial x}+\frac{a^{2} \partial^{2} c}{2}+\ldots x^{2}}{c-a \frac{\partial c}{\partial x}+\frac{a^{2} \partial^{2} c}{2}+\ldots x^{2}+\cdots}\right)_{x=E}
$$

$o$, trascurando $\mathrm{i}$ termini in grado superiore al secondo in $a$,

$$
p=1+\left[2 \frac{a}{c} \frac{\partial c}{\partial x}+\frac{a^{2}}{c^{2}}\left(\frac{\partial c}{\partial a}\right)^{2}\right]_{x=\Xi}
$$

Dalla (1) viene subito

$$
\left(\frac{1}{c} \frac{\partial c}{\partial x}\right)_{x=\Xi}=\frac{1}{\Xi}-\frac{\Xi}{2 k^{2} t}
$$

e dunque la condizione

$$
\frac{\partial \rho}{\partial t}=0
$$

si trasforma nell'altra

$$
\frac{\partial}{\partial t}\left[2\left(\frac{1}{\Xi}-\frac{\Xi}{2 k^{2} t}\right)+a\left(\frac{1}{\Xi}-\frac{2 k^{2} t}{\Xi}\right)^{2}\right]=0
$$

vale a dire

$$
2 k^{2}(a+\Xi) \Theta=a \Xi^{2}
$$

nella quale ultima si è indicato con $\Theta$ l'istante che corrisponde al massimo della $f$. e. m.

La (2) fornisce

$$
k^{2}=\frac{a \Xi^{2}}{2(a+\Xi) \Theta}
$$

2. Ho eseguito la misura di $\theta$ nel seguente modo: Feci tornire internamente un tubo di vetro alto poco più di 10 centimetri, aperto alle due estremita, in modo che a un'altezza fissa (per es. un centimetro) cominciasse una differenza nello 
spessore della parete. Un elettrodo foggiato a disco, introdotto per la parte superiore, veniva cosi a poggiare cogli orli sullo scalino che ne risultava.

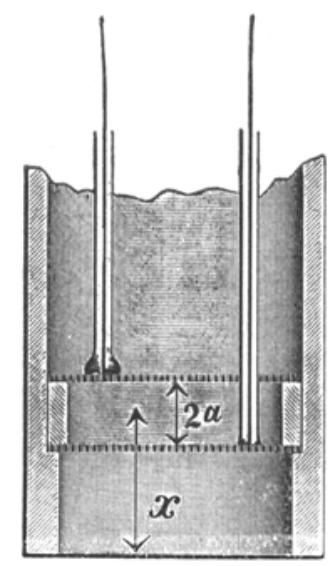

Questo elettrodo era costituito di un dischetto sottile di argento purissimo (Haereaus), piano e munito di numerosissimi forellini, ricoperto, prima dell' esperienza, mediante elettrolisi di $\mathrm{HCl}$ diluito, di uno strato ben uniforme di $\mathrm{AgCl}$. Sopra di esso si adattava un anello di vetro di altezza rigorosamente uniforme, e poi veniva un secondo elettrodo identico in tutto al primo. I fili di argento saldati a questi elettrodi venivano introdotti in tubi di vetro che erano fissati poi agli elettrodi stessi mediante un mastice isolante. Quello inferioro passava attraverso un foro dell'altro.

Il cilindro si riempiva dapprima di gelatina e poi vi si immergevano gli elettrodi, così preparati, quando questa era ancora fluida, avendo cura di evitare le bolle d'aria che facilmente rimangono incluse.

La gelatina era quella che usano le fabbriche di lastre fotografiche. Veniva ben lavata e poi posta a bagno maria a circa $40^{\circ}$ con tanta acqua da avere il colloide al $6 \%$; ottenutolo, si filtrava colla pompa e, dopo raffreddamento, avvenuta la solidificazione, si tagliava in pezzetti minuti e si sottoponeva a una corrente di acqua distillata. Prolungando sufficien- 
temente la dialisi si riesce ad avere il gel praticamente puro: per ciascuna preparazione si verificava che non era apprezzabile la f. e. m. fra due elettrodi di argento, ricoperti di $\mathrm{AgCl}$, immersi in un cilindro (precedemente descritto) ripieno di questa gelatina o posto per molte ore sull'acqua distillata corrente.

Per riempire bene il tubo rappresentato dalla figura, questo era posto sopra una lastrina di vetro ben piana e vi si versava il colloide appena fuso. Introdotti gli elettrodi e l'anello di vetro, appena la gelatina era sólidificata completamente, si rovesciava e si bagnava con acqua calda la lastrina dalla parte esterna in modo che potesse facilmente staccarsi. Ia superficie terminale inferiore risultava così senz'altro un piano. Appeso il cilindro a un supporto, si poneva sopra un recipiente in cui scorreva, mediante un sifone regolabile, la soluzione da studiarsi in modo che il liquido sfiorasse appena il piano di base. Gli effetti della gravità non vengono cosi a disturbare il processo diffusivo.

Trascorso il tempo $\Theta$, si portava rapidamente l'apparecchio sopra un altro recipiente dove scorreva l'acqua distillata. E allora si cominciava a fare la determinazione delle f. $m$. e. col metodo di compensazione adoperando una pila campione Clark e un galvanometro Hartmann e Braun $\left[1^{\circ}=7.250^{-6}\right.$ amp.; Res. $185 \mathrm{ohm}$. Le letture si facerano ogni sei minuti, e questo intervallo di tempo è sufficiente per apprezzare la differenza, benchè piccola, fra due misure consecutive, meno che negli istanti vicini a quello in cui si raggiunge il massimo. Per fissare nettamente il valore di $\Theta$ non c'è da far altro che aggiungere al valore del tempo per cui il massimo è raggiunto, la metà di quello durante il quale la f.e.m. pare mantenersi costante.

Nella seguente tabella sono riassunti i risultati delle esperienze. 


\begin{tabular}{|c|c|c|c|c|}
\hline \multicolumn{5}{|c|}{ Soluzioni di $\left.\mathrm{KCl}^{1}\right)$} \\
\hline \multirow{2}{*}{\multicolumn{2}{|c|}{$\begin{array}{l}{[\Xi=1,25(\mathrm{~cm})} \\
\text { concentrazione temperatura } \\
\text { molecolare media }\end{array}$}} & \multicolumn{3}{|c|}{$2 a=0,50$ (cm.)] } \\
\hline & & $\begin{array}{c}\theta \\
\text { (ore) }\end{array}$ & $\begin{array}{c}\theta \\
\text { (ore) }\end{array}$ & $\begin{array}{c}h^{2} \\
\mathrm{~cm}^{2} / \text { giorno }\end{array}$ \\
\hline \multirow{3}{*}{2,5} & $13, \overline{0}$ & 0.7 & 2,65 & 1,18 \\
\hline & 12,2 & 0,6 & $2, \check{3} 3$ & 1,22 \\
\hline & 14,0 & 0,4 & 2,60 & 1,19 \\
\hline \multirow{3}{*}{ 1,25 } & $13, \tilde{0}$ & 0,7 & 2,66 & 1,17 \\
\hline & 14,0 & 0,6 & 2,ธ̄อ̄ & 1,22 \\
\hline & 13,0 & $0, \overline{0}$ & 2,80 & 1,11 \\
\hline \multicolumn{5}{|c|}{ Soluzioni di $\mathrm{Na} \mathrm{Cl}^{2}$ ) } \\
\hline \multirow{2}{*}{\multicolumn{2}{|c|}{$\begin{array}{l}\qquad E=125(\mathrm{~cm}) \\
\text { concentrazione temperatura } \\
\text { molecolare media }\end{array}$}} & \multicolumn{3}{|c|}{$2 a=0, \tilde{0} 0(\mathrm{~cm})]}$. \\
\hline & & $\begin{array}{c}\theta \\
\text { (ore) }\end{array}$ & $\begin{array}{c}\boldsymbol{\theta} \\
\text { (ore) }\end{array}$ & $\begin{array}{c}k^{2} \\
\mathrm{~cm}^{2} \text { /giorno }\end{array}$ \\
\hline \multirow{3}{*}{2,0} & 12,0 & 0,7 & $3, \overline{5} 5$ & 0,88 \\
\hline & 13,2 & 0,6 & $3, \check{0} 0$ & 0,89 \\
\hline & $12, \tilde{0}$ & $0, \tilde{o}$ & 3,60 & 0,87 \\
\hline \multirow{3}{*}{1,0} & 14,0 & 0,7 & 3,50 & 0,89 \\
\hline & 12,5 & 0,6 & 3,43 & 0,91 \\
\hline & 13,1 & $0, \tilde{\jmath}$ & 3,45 & 0,90 \\
\hline
\end{tabular}

Se ora si riducono a $18^{\circ} \mathrm{i}$ valori di $k^{9}$ che figurano nell'ultima colonna, pensando che è legittimo ammettere che le mobilità degli ioni e la pressione osmotica dei sali disciolti abbiano nella gelatina adoperata lo stesso coefficiente di temperatura che nell'acqua ${ }^{3}$ ), si ottengono i valori medî :

$$
\begin{array}{lll}
1,33 & \text { per } & \mathrm{KCl} \\
1,01 & \text { per } & \mathrm{NaCl}
\end{array}
$$

1) I sali erano purissimi e provenivano dalla fabbrica Merck.

2) I sali erano purissimi e provenivano dalla fabbrica Merck.

3) Arrhenius. Lehrbuch der Elektronhemie (1901), pag. 148. 
Le misure di Oeholm '), che sono le più apprezzate, danno per $k^{2}$, in acqua, a $18^{\circ}$, i seguenti valori :

$\begin{array}{cc} & \mathrm{KCl} \\ \text { Cone. mol. } & k^{2} \\ 1,20 & 1,33 \\ 3,10 & 1,34 \\ & \mathrm{Na} \mathrm{Cl} \\ \text { Cone. mol. } & l^{2} \\ 1,45 & 1,07 \\ 2,80 & 1,06\end{array}$

L'esperienza descritta conduce a buoni risultati solo quando $\theta$ sia sufficientemente piccolo. Un criterio sperimentale per fissarne i limiti è il seguente: la soluzione deve circolare sotto la gelatina per un tempo cosi breve che quando la concentrazione del piano di base si riporta allo zero, la f. e. m. sia ancora nulla, e cioè sia nulla la concentrazione agli elettrodi o, per lo meno, all'elettrodo superiore.

1) Zeit. t. phys. C. 50, 308. 